\title{
Long acting nifedipine was as effective as hydrochlorothiazide plus amiloride for reducing mortality and morbidity in hypertension
}

\author{
Brown MJ, Palmer CR, Castaigne A, et al. Morbidity and mortality in patients randomised to double-blind treatment with a \\ long-acting calcium-channel blocker or diuretic in the International Nifedipine GITS study: Intervention as a Goal in \\ Hypertension Treatment (INSIGHT). Lancet 2000 Jul 29;356:366-72.

\begin{abstract}
QUESTION: In patients with hypertension who are at high risk for cardiovascular (CV) events, is long acting nifedipine, a calcium channel blocker, as effective as co-amilozide (hydrochlorothiazide and amiloride) for preventing CV and cerebrovascular mortality and morbidity?
\end{abstract}

\section{Design}

Randomised (allocation concealed*), blinded (patients, physicians, and outcome assessors),* placebo controlled trial with $\geqslant 3$ years follow up (Intervention as a Goal in Hypertension Treatment [INSIGHT] trial).

Setting

703 centres in 8 countries in western Europe and Israel.

\section{Patients}

7343 patients with hypertension were enrolled, 6575 were randomised, and 6321 (mean age 65 y, 54\% women) were studied after exclusion of 9 centres because of protocol violations. Patients were required to have $\geqslant 1$ additional $\mathrm{CV}$ risk factor (hypercholesterolaemia, smoking, family history of early myocardial infarction [MI], left ventricular hypertrophy or strain, coronary artery disease, or peripheral vascular disease). Follow up was 94\%.

Source of funding:

Bayer AG.

For correspondence: Professor M J Brown,

Clinical Pharmacology

Unit, Level 6, ACCI,

Box 110 ,

Addenbrooke's

Hospital, Cambridge

CB2 2QQ, UK. Fax

$+44(0) 1223762576$

\section{Intervention}

3157 patients were analysed in the long acting nifedipine group $(30 \mathrm{mg} / \mathrm{d})$, and 3164 were in the co-amilozide group (hydrochlorothiazide, $25 \mathrm{mg} / \mathrm{d}$, and amiloride, $2.5 \mathrm{mg} / \mathrm{d}$ ). If hypertension persisted, the regimen was intensified in steps that included doubling the dose of the study drug; adding atenolol, $25 \mathrm{mg}$ /day, or enalapril, $5 \mathrm{mg}$ /day; doubling the dose of the second drug; and adding another antihypertensive drug other than a calcium channel blocker or diuretic.

\section{Main outcome measures}

A composite end point of CV or cerebrovascular death and non-fatal MI, stroke, and heart failure. Secondary outcomes were all cause mortality, vascular death, and non-fatal vascular events.

\section{Main results}

Blood pressure (BP) decreased in both groups to approximately 138/82 mm Hg. Non-fatal heart failure was more common in the nifedipine group than in the co-amilozide group $(0.8 \% v 0.3 \%, \mathrm{p}=0.028)$ as were fatal MI $(0.5 \%$ v $0.2 \%, \mathrm{p}=0.017)$ and all adverse events $(49 \% v 42 \%, \mathrm{p}<0.001)$. Withdrawals because of adverse effects were higher in the nifedipine group than in the co-amilozide group $(23 \% v 16 \%, \mathrm{p}<0.001)$. The groups did not differ for the primary outcomes, including the composite end point $(6.3 \%$ in the nifedipine group $v$ $5.8 \%$ in the co-amilozide group, $\mathrm{p}=0.35$ ), all cause $(p=0.95)$ or cause specific mortality $(p \geqslant 0.14)$, non-fatal MI $(p=0.52)$, sudden death $(p=0.43)$, stroke $(p \geqslant 0.52)$, fatal heart failure $(p=0.63)$, or non-fatal $\mathrm{CV}$ events $(p \geqslant 0.1)$.

\section{Conclusions}

Nifedipine and co-amilozide were equally effective for preventing cardiovascular and cerebrovascular mortality and morbidity in high risk patients with hypertension. Fewer patients, however, tolerated nifedipine.

*See glossary.

\section{COMMENTARY}

INSIGHT and NORDIL add to the acronymic litany of recent trials addressing pharmacotherapy of hypertension-this litany consists of multiple trials with multiple comparisons spawned by multiple drugs from multiple classes with multiple actions. With the addition of the INSIGHT trial and NORDIL study, we have 10 completed long term trials that compare calcium antagonists with other antihypertensive drugs.

Such trials that evaluate morbidity and mortality effects of calcium antagonists have variable findings that are difficult to interpret:

- None was large enough to reliably detect moderate differences (10\% to $15 \%)$ in such clinically important outcomes as MI

- $\mathrm{BP}$ control with monotherapy was not achieved in as many as one third to one half of trial participants, depending on baseline and target BP concentrations

- Comparisons among agents were usually complicated by the addition of second and third drugs

- In large trials, such as INSIGHT and the Swedish Trial in Old Patients (STOP-2), ${ }^{2}$ a third or more of the participants were withdrawn from their initially assigned regimens because of adverse effects, difficulty with adherence to long term treatment, or both

- Occasional findings within trials, such as more fatal MI and non-fatal heart failure with calcium antagonists in the INSIGHT trial and fewer strokes with calcium antagonists in the NORDIL study, may result from chance because several comparisons were usually done

- Intermediate and long acting non-dihydropyridine and dihydropyridine calcium antagonists were being evaluated. Clinicians are rightfully wary of generalising beneficial and harmful class effects across these agents 


\title{
Diltiazem was as effective as diuretics or $\beta$ blockers, or both, at preventing cardiovascular mortality and morbidity
}

\author{
Hansson L, Hedner T, Lund-Johansen P, et al, for the NORDIL Study Group. Randomised trial of effects of calcium \\ antagonists compared with diuretics and b-blockers on cardiovascular morbidity and mortality in hypertension: the \\ Nordic Diltiazem (NORDIL) study. Lancet 2000 Jul 29;356:359-65. \\ QUESTION: In middle aged patients with hypertension, is diltiazem, a \\ non-dihydropyridine calcium antagonist, as effective as diuretics, $\beta$ blockers, or both at \\ preventing cardiovascular (CV) events?
}

\section{Design}

Randomised (allocation concealed*), blinded (outcome assessors),* controlled trial with mean follow up of 4.5 years (Nordic Diltiazem [NORDIL] study).

\section{Setting}

1032 primary healthcare centres in Norway and Sweden.

\section{Patients}

10881 patients (mean age $60 \mathrm{y}, 51 \%$ women) who had hypertension (diastolic blood pressure $[\mathrm{BP}] \geqslant 100 \mathrm{~mm}$ $\mathrm{Hg}$ on 2 occasions) and were aged 50 to 69 years (extended to 74 y during the study). Follow up was $>99 \%$.

\section{Intervention}

Patients were allocated to diltiazem $(n=5410)$ or to diuretics or $\beta$ blockers, or both $(n=5471)$. If hypertension persisted, the regimen was intensified. Diltiazem was started at 180 to $360 \mathrm{mg}$ /day, with stepped addition of an angiotensin converting enzyme (ACE) inhibitor, a diuretic or $\alpha$ blocker, and any other antihypertensive drug. In the other group, a thiazide diuretic or $\beta$ blocker was started with stepped addition of the other drug, an ACE inhibitor or $\alpha$ blocker, and any other antihypertensive drug except a calcium antagonist.

\section{Main outcome measures}

$\mathrm{BP}$ and combined fatal and non-fatal stroke, fatal and non-fatal myocardial infarction (MI), and other CV death.

\section{Main results}

Mean BP decreased in both groups, to $155 / 89$ in the diltiazem group and to $152 / 89 \mathrm{~mm} \mathrm{Hg}$ in the diuretics and $\beta$ blocker group. For patients who remained in the study for $\geqslant 24$ months, decreases in systolic but not diastolic BP were smaller in the diltiazem group than in the diuretics and $\beta$ blocker group (20/19 v 23/19 mm $\mathrm{Hg}, \mathrm{p}<0.001)$. The groups did not differ for the combined primary end point (16.6 $v 16.2$ events/1000 patient years, $\mathrm{p}=0.97)$, all $\mathrm{MI}(7.4 v 6.3, \mathrm{p}=0.17), \mathrm{CV}$ death (5.2 $v 4.5, \mathrm{p}=0.41)$, all cause mortality $(9.2 v 9.0$, $\mathrm{p}=0.99)$, all cardiac events (20.2 $v$ 19.2, $\mathrm{p}=0.57)$, diabetes mellitus (9.4 $v 10.8, \mathrm{p}=0.14)$, and congestive heart failure $(2.5 v 2.1, \mathrm{p}=0.42)$. Patients in the diltiazem group had fewer strokes than patients in the diuretics and $\beta$ blocker group (6.4 $v 7.9$ events/1000 patientyears, $\mathrm{p}=0.04$ ). The rate of adverse effects was similar among the groups.

\section{Conclusion}

Diltiazem was as effective as diuretics, $\beta$ blockers, or both for preventing the combined end point of stroke, myocardial infarction, and other cardiovascular death.

*See glossary.

COMMENTARY — continued from previous page

- Calcium antagonists are being compared with different agents within different classes. Clinicians realise that simple conclusions about calcium antagonists compared with "all other" agents are unlikely

- Some trials, such as NORDIL, do not achieve equivalence in BP lowering among calcium antagonists and other agents.

Given these complexities, we clinicians can embrace the following "truths." We have no strong, consistent evidence that intermediate or long acting calcium antagonists are superior or inferior to other antihypertensive agents in reducing CV disease and mortality. We have suggestive worrisome evidence that some intermediate and long acting calcium antagonists may increase such cardiac harms as MI and heart failure more than do diuretics (INSIGHT trial) or ACE inhibitors. ${ }^{2-5}$ We know that adverse effects of antihypertensive treatments vary. For example, peripheral edema is reported by as many as $25 \%$ of people taking calcium antagonists (INSIGHT trial), ${ }^{2}$ and cough is reported by as many as $30 \%$ of those taking ACE inhibitors. ${ }^{2}$ We know calcium antagonists are often more expensive than other anti-hypertensive agents. While we await results of additional large, long term trials, many evidence-based clinicians will continue to choose agents other than calcium antagonists as firstline treatment for patients with hypertension.

Cynthia D Mulrow, MD, MSc Audie L Murphy Memorial Veterans Hospital San Antonio, Texas, USA

1 MacMahon S, Neal B. Differences between blood-pressure-lowering drugs. Lancet 2000;356:352-3.

2 Hansson L, Lindholm LH, Ekbom T, et al. Randomised trial of old and new antihypertensive drugs in elderly patients: cardiovascular mortality and morbidity in the Swedish Trial in Old Patients with Hypertension-2 study. Lancet 1999;354:1751-6.

3 Tatti P, Pahor M, Byington RP, et al. Outcome results of the fosinopril versus amlodipine cardiovascular events randomized trial (FACET) in patients with hypertension and NIDDM. Diabetes Care 1998;21:597-603.

4 Estacio RO, Jeffers BW, Hiatt WR, et al. The effect of nisoldipine as compared with enalapril on cardiovascular outcomes in patients with non-insulin-dependent diabetes and hypertension. N Engl J Med 1998;338:645-52.

5 Casiglia E, Spolaore P, Mazza A, et al. Effect of two different therapeutic approaches on total and cardiovascular mortality in a cardiovascular study in the elderly (CASTEL).Jpn Heart J 1994;35:589-600. 